\title{
Highly Pathogenic Strains of Escherichia coli Revealed by the Distinct Electrophoretic Patterns of Carboxylesterase B
}

\author{
By Ph. GOULLET* AND B. PICARD \\ Laboratoire de Microbiologie, Faculté de Médecine Xavier-Bichat (Université Paris VII), \\ 16 rue Henri Huchard, 75018 Paris, France
}

(Received 4 October 1985; revised 14 February 1986)

\begin{abstract}
One hundred and ninety one strains of Escherichia coli isolated from extra-intestinal infections and 85 strains isolated from the stools of healthy human beings were compared for electrophoretic mobility and isoelectric point of carboxylesterase $\mathbf{B}$, and for production of $\alpha$-haemolysin and the presence of mannose resistant haemagglutinin. Fast and slow electrophoretic mobilities were distinguished among the strains. The frequency of strains showing slow mobilities was considerably higher when they originated from extra-intestinal infections $(40 \%)$ than when they were obtained from the stools of healthy individuals $(7 \%)$. In a two-dimensional electrophoretic profile, the fast and slow mobility variants of carboxylesterase $B$ were resolved into two patterns, $B_{1}$ and $B_{2}$, respectively. The frequency of pathogenic strains that concomitantly produced $\alpha$-haemolysin and mannose resistant haemagglutinin was $48.7 \%$ for strains of pattern $B_{2}$ but only $2.8 \%$ for strains of pattern $B_{1}$. Thus, the electrophoretic pattern $B_{2}$ of carboxylesterase $\mathrm{B}$ appears to be a molecular marker for a group of highly pathogenic $E$. coli strains which are frequently implicated in extra-intestinal infections.
\end{abstract}

\section{INTRODUCTION}

In the preceding paper (Goullet \& Picard, 1986) we showed by conventional electrophoresis (CE) that Escherichia coli strains producing slow mobility variants of carboxylesterase B were more frequently isolated from extra-intestinal infections than from human stools.

To substantiate this electrophoretic discrimination between commensal and pathogenic strains of $E$. coli, isoelectrofocusing (IEF) was used with CE to establish two-dimensional electrophoretic profiles (Goullet \& Picard, 1985a, b; Picard \& Goullet, 1985) of carboxylesterase B. These data correlate with production of $\alpha$-haemolysin (Smith, 1963; van den Bosch et al., 1981; Hughes et al., 1983; Cavalieri et al., 1984) and the presence of a mannose resistant haemagglutinin (MRHA) (Evans et al., 1980; Vaisanen et al., 1981). These are believed to be virulence factors of invasive bacterial strains (Hagberg et al., 1981; Welch et al., 1981; van den Bosch et al., 1981, 1982; Cavalieri \& Snyder, 1982; Gadeberg et al., 1983; Hacker et al., 1983). Using a random collection of isolates obtained from extra-intestinal infections and the faeces of healthy human beings, highly pathogenic strains were delineated by a distinct electrophoretic pattern of the esterase and by the frequent production of $\alpha$-haemolysin and a MRHA.

\section{METHODS}

Sources of the bacterial strains. Commensal strains of Escherichia coli were obtained from faecal samples collected in Paris from 85 healthy human beings. Pathogenic strains of E. coli were recovered during the acute phase of 191 cases of extra-intestinal infections. Essentially the patients were grouped as follows: (i) two groups of hospitalized subjects (81 patients at St Antoine Hospital, Paris and 66 patients at Durance Hospital, Avignon) and (ii) one

Abbreviations: CE, conventional electrophoresis; HR, haemolysin release; IEF, isoelectrofocusing; MRHA, mannose resistant haemagglutinin; $M_{F}$, electrophoretic mobility. 
group of 44 non-hospitalized patients who were treated by general practitioners in Paris. To avoid bias, strains from each category were taken randomly without considering the nature of the infection. Thus, 160 of the 191 pathogenic strains $(83.5 \%)$ were derived from urinary tract infection as confirmed by the presence of significant bacteriuria ( $>10^{5}$ bacteria $\mathrm{ml}^{-1}$ ) determined using a calibrated loop (Isenberg et al., 1985). Of the remaining organisms, 17 strains $(9 \%)$ were obtained from cases of septicaemia and 14 strains $(7.5 \%)$ were from other miscellaneous infections, including abcesses, lung infections, cholecystitis and some surgical infections. In all cases, the strains were obtained in pure culture by isolation on tryptic soy agar.

Electrophoretic analysis. Growth conditions, preparation of extracts, protein estimation, horizontal slab PAGE, estimation of electrophoretic mobility $\left(M_{F}\right.$ value), polyacrylamide gel isoelectrofocusing, determination of isoelectric points and esterase staining were described by Goullet \& Picard (1985a).

$\alpha$-Haemolysin assays. $\alpha$-Haemolysin activity was routinely detected using horse erythrocyte agar $(2 \%, v / v$, erythrocytes) (Le Minor \& Le Coueffic, 1975) and was subsequently confirmed by titration of $\alpha$-haemolytic activity (van den Bosch et al., 1980).

During the exponential phase of growth in fresh alkaline veal meat broth (Smith, 1963), supernatants were filtered and diluted from $1: 2$ to $1: 256$ in Tris buffered saline $(0 \cdot 01 \mathrm{M}-\mathrm{Tris} / \mathrm{HCl}, 0 \cdot 135 \mathrm{M}-\mathrm{NaCl}, \mathrm{pH} 7 \cdot 5)($ van den Bosch et al., 1980). Fresh sheep erythrocytes, washed three times and suspended to $2 \%$ in Tris buffered saline, were then added. The mixtures were incubated and centrifuged. Haemolysin release (HR) was measured with a Beckman spectrophotometer (model 24) at $540 \mathrm{~nm}$. The titre was estimated as the highest dilution giving an HR of at least $25 \%$ of the maximal HR of the erythrocytes.

MRHA assays. These were done on glass microscope slides using type A human erythrocytes (Vosti, 1979) that had been washed three times and resuspended to $3 \%$ in phosphate buffered saline $\left(0.005 \mathrm{M}-\mathrm{KH}_{2} \mathrm{PO}_{4}, 0.032 \mathrm{M}-\right.$ $\mathrm{Na}_{2} \mathrm{HPO}_{4}, 0.170 \mathrm{M}-\mathrm{NaCl}, 0.010 \mathrm{M}-\mathrm{KCl}, \mathrm{pH} 7 \cdot 2$ ) containing $1 \%$ (w/v) methyl $\alpha$-D-mannopyranoside (Sigma). Bacteria grown on agar were mixed with a drop $(50 \mu \mathrm{l})$ of the erythrocyte suspension at room temperature. Agglutination was read after agitation for about $1 \mathrm{~min}$, and was compared with positive and negative controls.

\section{RESULTS \\ $M_{F}$ distribution}

The $M_{F}$ distribution patterns of carboxylesterase B produced by commensal and pathogenic strains of $E$. coli are shown in Fig. 1. For both commensal and pathogenic strains, fast (ranging from $M_{F} \simeq 74$ to $M_{F} \simeq 66$ ) and slow (ranging from $M_{F} \simeq 63$ to $M_{F} \simeq 57$ ) electrophoretic mobilities may be distinguished. The two types of mobilities were not simultaneously observed in the same strain. Most $(90.5 \%)$ of the commensal strains showed fast mobilities; $7 \%$ showed slow mobilities and $2.5 \%$ (two strains) produced no detectable carboxylesterase B activity. For pathogenic strains, $56.5 \%$ of the isolates showed fast mobilities which were distributed around $M_{F} \simeq 70 ; 40 \%$ showed slow mobilities and $3.5 \%$ (seven strains) produced no detectable carboxylesterase B activity.

\section{pI distribution}

The pI distribution of carboxylesterase B produced by commensal strains and by pathogenic strains is shown in Fig. 2. For both types, the pI values of the esterase ranged from 4.5 to 5 . In general the proportion of strains with pI values from 4.8 to 5 was higher for pathogenic isolates $(45.5 \%)$ than for commensal isolates $(18.8 \%)$.

\section{Two-dimensional electrophoretic profile distribution}

Two-dimensional electrophoretic profiles defined by mobility and the pI of each electrophoretic variant of carboxylesterase $B$ as produced by commensal and pathogenic strains are shown in Fig. 3. Several mobility variants occurred for a single pI, and conversely, several pI variants were recovered in a single mobility. The number of electrophoretic variants resolved by two-dimensional electrophoretic profiles was higher than the number of electrophoretic variants resolved by CE or by IEF. Thus CE, IEF and two-dimensional electrophoretic profiles detected 7,6 and 13 electrophoretic variants for commensal strains and 11, 8 and 26 electrophoretic variants for pathogenic strains, respectively.

The electrophoretic variants may be classified according to two distinct electrophoretic patterns, namely $B_{1}$, which was defined by mobilities ranging from $M_{F} \simeq 74$ to $M_{F} \simeq 66$ and by 


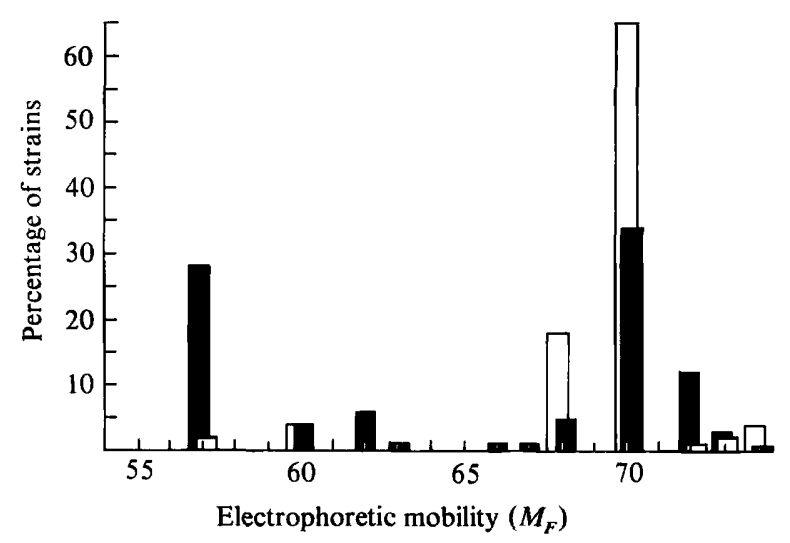

Fig. 1. Histograms of the electrophoretic mobility distribution of carboxylesterase B. $\square$, Commensal strains; $\mathbf{Z}$, pathogenic strains.

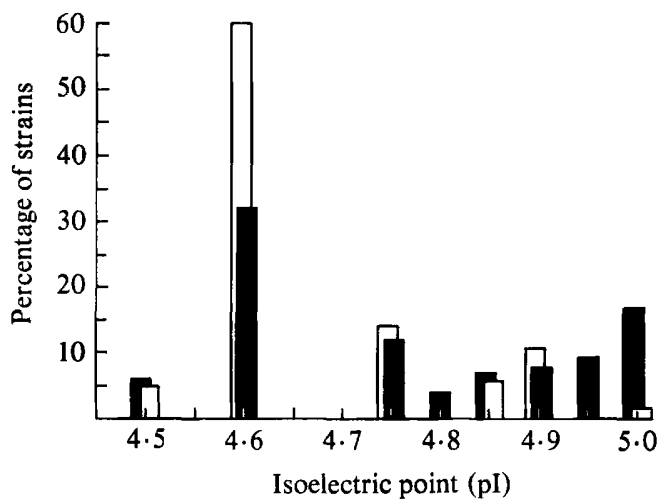

Fig. 2. Histograms of the isoelectric point distribution of carboxylesterase B. $\square$, Commensal strains; a, pathogenic strains.

pI ranging from 4.5 to 4.9 , and $\mathrm{B}_{2}$, which was defined by mobilities ranging from $M_{F} \simeq 63$ to $M_{F} \simeq 57$ and by $\mathrm{pI}$ ranging from 4.8 to 5 . The frequency of strains showing the electrophoretic variant $M_{F} \simeq 57$ and pI 5 was higher among pathogenic isolates $(17.3 \%)$ than among commensal isolates $(2.3 \%)$. Moreover, among the strains producing detectable carboxylesterase $B$ activity, 77 commensal isolates showed electrophoretic pattern $B_{1}$ (these isolates were designated as $\mathbf{C B}_{1}$ ) but only six commensals showed electrophoretic pattern $\mathbf{B}_{2}$ (these isolates were designated as $\mathrm{CB}_{2}$ ). In contrast, 108 pathogenic isolates $(56.5 \%)$ showed electrophoretic pattern $B_{1}$ (these strains were designated as $P B_{1}$ ) and 76 of the pathogens $(40 \%)$ showed electrophoretic pattern $B_{2}$ (these isolates were designated as $P_{2}$ ).

Strains with the electrophoretic pattern $B_{2}$ (Fig. 3) were isolated from all three groups of patients, and from the diverse extra-intestinal infections. However, the frequency of $\mathbf{P B}_{2}$ strains was slightly higher among the organisms isolated from the two groups of hospitalized patients $(44.5 \%$ and $41 \%$, respectively) than among those derived from non-hospitalized patients $(29.5 \%)$. This also applied to strains derived from cases of septicaemia and other extra-urinary infections $(48.5 \%)$ as compared with strains from urinary tract infections $(38 \%)$.

Correlation between electrophoretic pattern and production of $\alpha$-haemolysin and MRHA

The production of $\alpha$-haemolysin and/or the presence of MRHA was much higher in pathogenic than in commensal strains (Table 1). The relationship between $\alpha$-haemolysin and MRHA and electrophoretic patterns $B_{1}$ and $B_{2}$ is shown in Table 2. In all cases, haemolysis was 


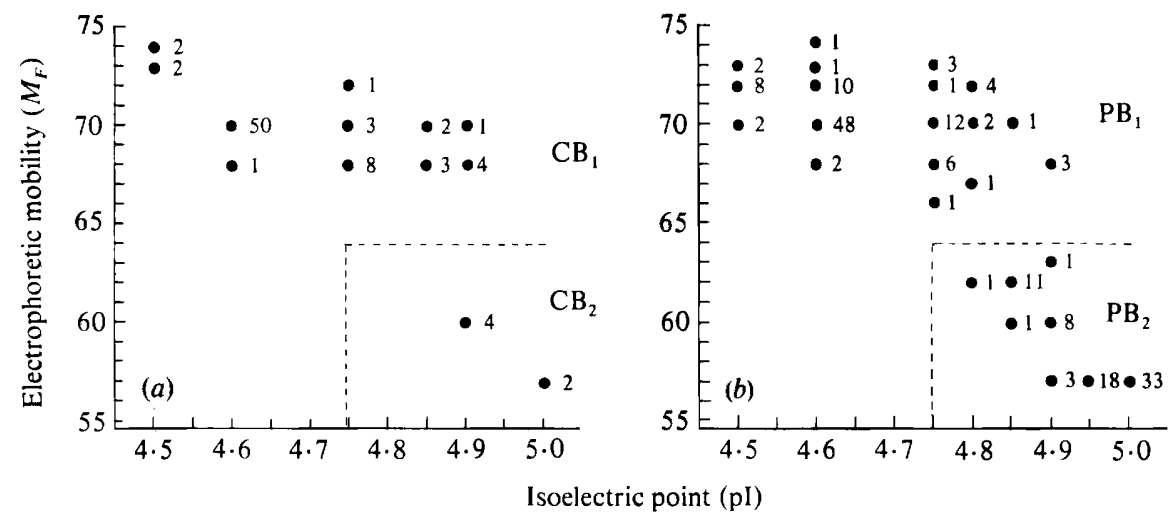

Fig. 3. Two-dimensional electrophoretic profile established by plotting electrophoretic mobility against isoelectric point of carboxylesterase B for 83 commensal strains $(a)$ and for 184 pathogenic strains $(b)$. The number of strains producing each electrophoretic variant is included.

Table 1. Production of $\alpha$-haemolysin and MRHA in commensal and pathogenic strains of $E$. coli

\section{Characteristic}

Producing $\alpha$-haemolysin without MRHA Producing MRHA without $\alpha$-haemolysin Producing concomitantly $\alpha$-haemolysin and MRHA Producing neither $\alpha$-haemolysin nor MRHA

\begin{tabular}{|c|c|}
\hline \multicolumn{2}{|c|}{ No. of strains } \\
\hline Commensal & Pathogenic \\
\hline $\begin{array}{r}2 \\
4 \\
2 \\
77\end{array}$ & $\begin{array}{l}14 \\
46 \\
41 \\
90\end{array}$ \\
\hline 85 & 191 \\
\hline
\end{tabular}

Table 2. Correlation between the two electrophoretic patterns of carboxylesterase $B$ and production of $\alpha$-haemolysin and MRHA in commensal and pathogenic strains of $E$. coli

For $\mathrm{CB}_{1}, \mathrm{CB}_{2}, \mathrm{~PB}_{1}$ and $\mathrm{PB}_{2}$ designations, see text.

\section{Characteristic}

Producing $\alpha$-haemolysin without MRHA

Producing MRHA without $\alpha$-haemolysin

Producing concomitantly $\alpha$-haemolysin and MRHA

Producing neither $\alpha$-haemolysin nor MRHA

\begin{tabular}{|c|c|c|c|}
\hline \multicolumn{4}{|c|}{ No. of strains } \\
\hline $\mathrm{CB}_{1}$ & $\mathrm{CB}_{2}$ & $\mathrm{~PB}_{1}$ & $\mathrm{~PB}_{2}$ \\
\hline 1 & 1 & 3 & 9 \\
\hline 3 & 1 & 29 & 17 \\
\hline 0 & 2 & 3 & 37 \\
\hline 73 & 2 & 73 & 13 \\
\hline 77 & 6 & 108 & 76 \\
\hline
\end{tabular}

confirmed by the production of $\alpha$-haemolysin in alkaline meat broth (titres were in the range of $1: 2$ to $1: 256$ ). The percentage of strains producing $\alpha$-haemolysin and/or MRHA was higher in group $\mathrm{PB}_{1}$ than in group $\mathrm{PB}_{2}$. In the case of strains producing MRHA the increase was about $2 \cdot 4$-fold; for strains producing $\alpha$-haemolysin the increase was 11-fold, and for strains producing $\alpha$-haemolysin and MRHA concomitantly the increase was $17 \cdot 6$-fold. Although the number of $\mathrm{CB}_{2}$ isolates (only six) was too low to allow statistical analysis of the data, it appeared that these strains were similar in the production of $\alpha$-haemolysin and MRHA to $\mathrm{PB}_{2}$ strains. Thus, the two commensal strains that concomitantly produced $\alpha$-haemolysin and MRHA, were both $\mathrm{CB}_{2}$ isolates. 


\section{DISCUSSION}

As in previous investigations on the diversity of bacterial esterases (Goullet \& Picard, 1985a, $b$; Picard \& Goullet, 1985), the number of electrophoretic variants of carboxylesterase B resolved by two-dimensional electrophoretic profiles was higher than the number of electrophoretic variants defined by CE or by IEF. Two distinct electrophoretic patterns - $\mathbf{B}_{1}$ (from $M_{F} \simeq 74$ to $M_{F} \simeq 66$ ) and $\mathrm{B}_{2}$ (from $M_{F} \simeq 63$ to $M_{F} \simeq 57$ ) were clearly defined by $\mathrm{CE}$ whereas IEF showed a continuous spectrum of $\mathrm{pI}$ variants from 4.75 to 5 (Fig. 3). The poorer discrimination obtained by IEF is due to the fact that several mobility variants of patterns $B_{1}$ and $B_{2}$ fall into a single $\mathrm{pI}$. Carboxylesterase $\mathbf{B}$ showing electrophoretic pattern $\mathbf{B}_{2}$ was more prevalent among clinical isolates from extra-intestinal infections $(40 \%)$ than in faecal isolates from healthy subjects $(7 \%)$.

Since the isolates were taken randomly from a variety of infections and sources, esterase electrophoretic pattern $\mathbf{B}_{2}$ may be considered as a molecular characteristic often associated with virulence. The production of $\alpha$-haemolysin and MRHA was much higher in isolates from extra-intestinal infections than in commensal isolates as previously reported (van den Bosch et al., 1982; Hagberg et al., 1981; Cavalieri et al., 1984). The electrophoretic pattern of carboxylesterase B correlates with the presence of $\alpha$-haemolysin and MRHA (Table 2) in two ways. Firstly, the proportion of isolates producing MRHA but not $\alpha$-haemolysin was six times higher in totally pathogenic strains $(24 \%)$ than in $\mathrm{CB}_{1}$ strains $(4 \%)$ [the percentage of isolates producing MRHA without $\alpha$-haemolysin was not significantly different in PB $_{1}(27 \%)$ and in $\mathrm{PB}_{2}(22.5 \%)$ strains]. Secondly, the proportion of isolates producing concomitantly $\alpha$-haemolysin and MRHA was 17.6 times greater in $\mathrm{PB}_{2}(48.68 \%)$ than in $\mathrm{PB}_{1}(2.77 \%)$ strains. The association of electrophoretic pattern $B_{2}$ with both clinical virulence and production of $\alpha$-haemolysin and MRHA suggests the suitability of using carboxylesterase B as a molecular marker for highly pathogenic organisms.

This work was supported by grants from the Conseil Scientifique de la Faculte Xavier Bichat (University Paris VII) and from the Conseil de la Ville de Paris.

\section{REFERENCES}

van den Bosch, J. F., Postma, P., De Graaff, J. \& MacLaren, D. M. (1980). Determination of the $\alpha$ hemolytic activity of haemolytic urinary Escherichia coli strains. FEMS Microbiology Letters 8, 75-77.

van den Bosch, J. F., Postma, P., De GraafF, J. \& MACLAREN, D. M. (1981). Haemolysis by urinary Escherichia coli and virulence in mice. Journal of Medical Microbiology 14, 321-331.

van den Bosch, J. F., Postma, P., Koopman, P. A. R., De Graaff, J., Maclaren, D. M., Van Brenk, D. G. \& GuineE, P. A. M. (1982). Virulence of urinary and faecal Escherichia coli in relation to serotype, haemolysis and haemagglutination. Journal of Hygiene 88, 567-577.

Cavalieri, S. J. \& SNyder, I. S. (1982). Cytotoxic activity of partially purified Escherichia coli alpha hemolysin. Journal of Medical Microbiology 15, 1121.

Cavalieri, S. J., Bohach, G. A. \& Snyder, I. S. (1984). Escherichia coli $\alpha$-haemolysin : characteristics and probable role in pathogenicity. Microbiological Reviews 48, 326-343.

Evans, D. J., Evans, D. G., Young, L. S. \& PitT, J. (1980). Hemagglutination typing of Escherichia coli: definition of seven hemagglutination types. Journal of Clinical Microbiology 12, 235-242.

GADEBERG, O. V., ØRSKov, I. \& RHODES, J. M. (1983).
Cytotoxic effect of an alpha-hemolytic Escherichia coli strain on human blood monocytes and granulocytes in vitro. Infection and Immunity 41, 358-364.

Goullet, Ph. \& PiCARD, B. (1985a). A two-dimensional electrophoretic profile for bacterial esterases. Electrophoresis 6, 132-135.

Goullet, Ph. \& Picard, B. (1985b). Etude du polymorphisme électrophoretique des lactate-, malate- et glutamate-deshydrogenases, de la phosphatase acide et des esterases de Providencia alcalifaciens, $P$. stuartii et $P$. rustigianii. Annales de l'Institut Pasteur/Microbiologie 136A, 347-358.

Goullet, Ph. \& PiCard, B. (1986). Comparative esterase electrophoretic polymorphism of Escherichia coli isolates obtained from animal and human sources. Journal of General Microbiology 132, 18431851.

Hacker, J., Hughes, C., Hof, H. \& Goebel, W. (1983). Cloned hemolysin genes from Escherichia coli that cause urinary tract infection determine different levels of toxicity in mice. Infection and Immunity $\mathbf{4 2 ,}$ 57-63.

Hagberg, L., Jodal, U., Korhonen, T. K., LidiNJANSON, G., LINDBERG, U. \& SVANBoRg-Eden, C. (1981). Adhesion, hemagglutination and virulence of Escherichia coli causing urinary tract infections. Infection and Immunity 31, 564-570. 
Hughes, C., Hacker, J., Roberts, A. \& Goebel, W. (1983). Hemolysin production as a virulence marker in symptomatic and asymptomatic urinary tract infections caused by Escherichia coli. Infection and Immunity 39, 546-551.

ISENBERG, H. D., Washington, J. A., II, Balows, A. \& SoNNENWIRTH, A. C. (1985). Collection, handling and processing of specimens. In Manual of Clinical Microbiology, 4th edn, pp. 73-98. Edited by E. H. Lennette. Washington, DC: American Society for Microbiology.

LE MinoR, S. \& Le CouefFic, E. (1975). Etude sur les hémolysines des Enterobacteriaceae. Annales de microbiologie 126, 313-332.

PiCard, B. \& Goullet, Ph. (1985). Comparative electrophoretic profiles of esterases, and of glutamate, lactate and malate dehydrogenases, from Aeromonas hydrophila, $A$, caviae and $A$. sobria. Journal of General Microbiology 131, 3385-3391.
SмIтн, H. W. (1963). The haemolysins of Escherichia coli. Journal of Pathology and Bacteriology 85, 197211.

Vaisanen, V., Tallgren, L. G., Makela, P. H., Kallenius, G., Hultberg, H., Elo, J., Sittonen, A., Svanborg-Eden, C., Svenson, S. B. \& KorHONEN, T. (1981). Mannose-resistance haemagglutination and $P$ antigen recognition are characteristic of Escherichia coli causing primary pyelonephritis. Lancet 26, 1366-1369.

Vosti, K. L. (1979). Relationship of hemagglutination to other biological properties of serologically classified isolates of Escherichia coli. Infection and Immunity 25, 507-512.

Welch, R. A., Dellinger, E. P., Minshew, B. \& Falkow, S. (1981). Haemolysin contributes to virulence of extra-intestinal $E$. coli infections. Nature, London 294, 665-667. 